\title{
Root Architecture Modeling and Visualization in Wheat
}

\author{
Liang Tang, Feng Tan, Haiyan Jiang, Xiaojun Lei, Weixing Cao, \\ and Yan Zhu*
}

\author{
Jiangsu Key Laboratory for Information Agriculture, Nanjing Agricultural University, \\ Nanjing, 210095, PR China \\ yanzhu@njau.edu.cn
}

\begin{abstract}
This paper aims to develop a root morphological model in wheat (Triticum aestivum L.), and the realistic visualization of root growth under different soil conditions in wheat. Based on the topology of the wheat root system, historical literature and experimental data, a root morphological model in wheat was developed preliminarily using thermal time as a driving factor, including sub-models of root emergence, root growth rate, and root axis curvature. Based on 3D visualization technology, a three-dimensional visualization model of the root axis in wheat was developed by using the platform of VC++.net and OpenGL library, including sub-models of geometry, texture mapping, and light rendering. Integrated the established root morphological model and the visualization model, the three-dimensional visualization of a root system in wheat under different soil conditions was realized. This study lays a foundation for further development of a visualization system for the whole wheat plant.
\end{abstract}

Keywords: Wheat; Root; Architecture; Model; Simulation; Visualization.

\section{Introduction}

Plant root systems play an important role in the development of new wheat germplasm with improved drought tolerance, nutrient and water uptake efficiencies and lodging resistance[1]. Root system development and structure are particularly difficult to study because of the difficulty of observing and quantifying the architecture of actual roots and the complexity and plasticity of roots as geometric objects [2].

Simulation models and computer visualization are useful tools to study root architecture. Several studies dedicated to the visualization of root system architecture have emerged during these last years, using different approaches. One approach is of fractal description [3-5], which uses statistical relationships between typical dimensions (e.g., length between branches, branching angles and diameters) observed locally throughout the root system. This approach, however, is essentially static because it does not rely on morphogenetic processes, thus rendering the models nearly unable to simulate architectures bearing developmental information. The approach

Corresponding author. 
based on development [2, 6-8] has been used more often. It formalizes and combines in a mathematical framework the main developmental rules involved in the dynamics of root system architecture. L systems [9-10] is another approach which has been used for root architecture modeling and visualization [11-12]. This approach links with a physiological model to simulate both plant function and structure, but it has not often been applied to the plant root. Greenlab is a functional-structural model of plant growth for environmental applications, examples have been applied to root system modeling [13, 14] and performing well, however visualization technologies like random processing and collision detection were not studied in detail.

Wheat (Triticum aestivum L.) is the most important grain crop in the world, more than half of world's population relies on wheat as its primary food staple. Existing studies on wheat root growth modeling and visualization emphasize either developmental processes or visualization technology. Thus, the objectives of this paper are to develop a 3D root morphological model in wheat based on historical literature and experimental data and realistic root growth visualization in wheat under different conditions by using 3D geometrical modeling techniques.

\section{Structure of Wheat Root System}

Two root types are distinguished in wheat: the seminal roots (also called primary roots), which develop at the scutellar and epiblast nodes of the embryonic hypocotyl of the germinating caryopsis; adventitious roots, which subsequently emerge from the coleoptilar nodes at the base of the apical culm and tillers [1]. These two categories of roots function in a complementary manner, and thus the root system must be considered as a whole. The number of primary roots in a cereal is usually five to seven, but may reach ten. Lateral roots (also called branch root) emerge on the primary roots and adventitious roots when roots grow until a certain period. First order and second order lateral roots also can grow on the primary roots and adventitious roots at a certain interval. Thus, the wheat root system is comprised of primary roots, adventitious roots and different orders of lateral roots in the soil (Fig. 1).

A binary tree structure was used to analyze the structure of wheat root system. The root system can be divided into different types of root axis, each root axis has several nodes, from each node can emerge two sub-trees. The root tip has no children nodes. These nodes include root growth information including root hierarchy and branch information (Fig. 2).

\section{Morphological Model Descriptions}

The root morphological model simulates the dynamic growth processes of the three dimensional structure of a root. The basic information needed to model the morphology of a single root is the number of roots, the times of root (primary roots, adventitious roots, and lateral roots) emergence, the growth rate of the root axis, and the growth direction of the root axis. Each root was thought of a collection of axial segments which change direction according to the morphological model. 


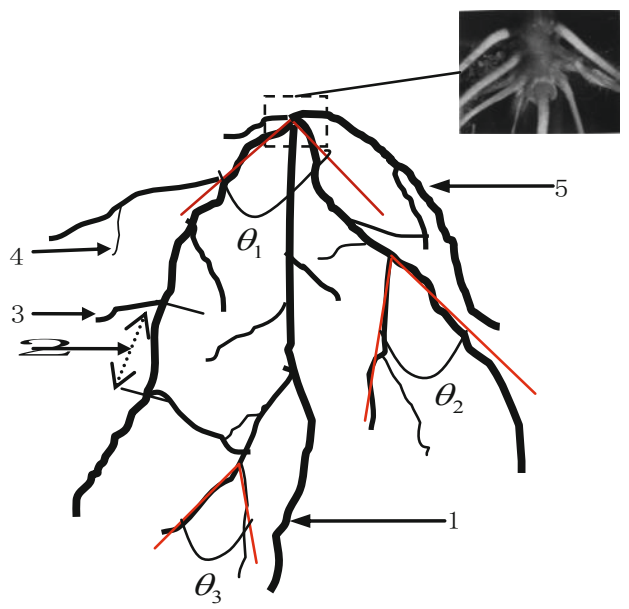

1. Primary root; 2. Branching interval; 3 . First order lateral root; 4 . Secondary order lateral root; 5. Adventitious root

Fig. 1. Root architecture in wheat

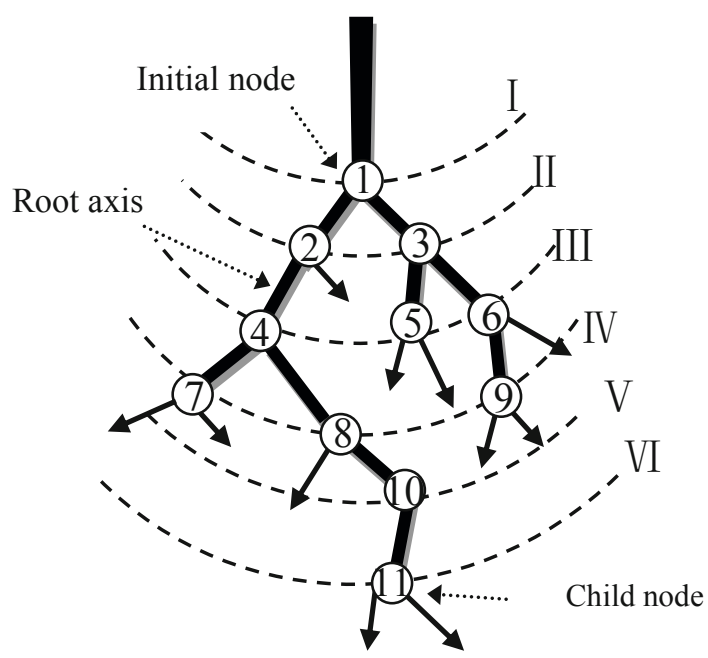

Fig. 2. Hierarchical structure of root nodes in wheat

\subsection{Root Emergence}

Primary root emergence. The primary roots emerge when wheat seed germinates, two or three pairs of primary roots emerge from below simultaneously, and the roots emerging on each node display radial symmetry [15]. The number of primary roots $(N)$ is significantly related to thermal time $\left(T T,{ }^{\circ} \mathrm{Cd}\right)$, as following:

$$
N=2.64 \cdot \ln (T T)-6.28 \text {. }
$$


Adventitious roots emergence. The growth of leaves, tillers and adventitious roots has a synchronous relationship: when leaf $n+3$ has emerged, a root emerges on leaf node $\mathrm{n}$ [16]. Integrating a previous study on the leaf phyllochron [17], the relationship between emergence of the adventitious root and thermal time was described by equation (2).

$$
T T_{a(b+2)}=\left\{\begin{array}{lr}
102+P H Y L L \times(b+1) & a=0 \\
102+P H Y L L \times(a+b+3) & 1 \leq a \leq S N
\end{array}\right.
$$

where $a$ is the tiller position where the adventitious root grows, and $b$ is the node position on the tiller where the root grows. $T T_{\mathrm{a}(\mathrm{b}+2)}$ is the thermal timewhen a root emerges on node $b$ of tiller $a\left({ }^{\circ} \mathrm{Cd}\right)$, PHYLL is phyllochron, 102 is the thermal time from sowing to emergence $\left({ }^{\circ} \mathrm{Cd}\right), S N$ is the number of effective tillers.

Lateral roots emergence. According to historical experimental data and the literature [18], the branching interval of main and first order lateral roots were $0.2 \mathrm{~mm}^{\circ} \mathrm{Cd}^{-1}$, and secondary order lateral roots were $0.05 \mathrm{~mm}^{\circ} \mathrm{Cd}^{-1}$. Branching can happen in the intervals. Branching probabilities were calculated by the interval of the adjacent two lateral roots.

The lateral root radius after branching was determined by a law which adopted by SimRoot [19], the section area before root branching was calculated by the section areas after root branching.

$$
d=\alpha \times\left(d_{1}^{2}+d_{2}^{2}\right)
$$

where $d$ is root diameter before branching $(\mathrm{mm}) ; d_{1}$ and $d_{2}$ are the lateral root diameters after branching $(\mathrm{mm}) ; \alpha$ is weighting coefficient, based on experimental data, taking an $\alpha$ value of 0.6 in this study.

\subsection{Root Growth Rate}

Different types of roots have different growth rates, the growth rate of a primary root can be several centimeters per day, while the growth rate of secondary order lateral root can be several millimeters per day. Roots grow rapidly when thermal time is less than $120^{\circ} \mathrm{Cd}$. Above $120^{\circ} \mathrm{Cd}$, root growth rate decreases. The growth rate of a single root was calculated as following:

When thermal time was less than $120{ }^{\circ} \mathrm{Cd}$, the growth rate $(\mathrm{V}(T T))$ of primary, adventitious and first order lateral root were:

$$
\mathrm{V}(T T)=-7 \times 10^{-6} T T^{2}+0.0375 T T-17 .
$$

The growth rate of secondary branch of root:

$$
\mathrm{V}(T T)=-1 \times 10^{-6} T T^{2}+0.0564 T T-47 \text {. }
$$

When thermal time was greater than $120{ }^{\circ} \mathrm{Cd}$, the growth rate of primary and adventitious root was:

$$
\mathrm{V}(T T)=2.1 \ln (T T)+5.2 .
$$

The growth rate of first order lateral root was: 


$$
\mathrm{V}(T T)=5.4 \ln (T T)-20 \text {. }
$$

The growth rate of secondary order lateral root was:

$$
\mathrm{V}(T T)=5.2 \ln (T T)-21 \text {. }
$$

\subsection{Growth Direction of Root}

Growth direction of primary and adventitious root axis. The method developed by Clausnitzer and Hopmans [20] was adopted in this study for the calculation of the growth direction of the root axis. The growth direction (D) is computed from three directional components: the initial direction $\left(\mathrm{D}_{-1}\right)$ of the root at the previous time step, geotropism $\left(\mathrm{D}_{\mathrm{G}}\right)$, mechanical constraint $\left(\mathrm{D}_{\mathrm{M}}\right)$ which may be considered as isotropic (random vector). The three vectors determine the final growth direction:

$$
\mathrm{D}=\mathrm{D}_{-1}+\mathrm{D}_{\mathrm{G}}+\mathrm{D}_{\mathrm{M}}
$$

The final position of root axis is rotated to an orthonormal coordinates, and can be denoted as following:

$$
\begin{aligned}
& \mathrm{X}_{2}=\mathrm{X}_{1}+\mathrm{L} \cdot \cos \alpha \\
& \mathrm{Y}_{2}=\mathrm{Y}_{1}+\mathrm{L} \cdot \cos \beta \\
& \mathrm{Z}_{2}=\mathrm{Z}_{1}+\mathrm{L} \cdot \cos \gamma
\end{aligned}
$$

$\left(\mathrm{X}_{1}, \mathrm{Y}_{1}, \mathrm{Z}_{1}\right)$ is initial growth position of the root axis in a growth cycle, $\left(\mathrm{X}_{2}, \mathrm{Y}_{2}, \mathrm{Z}_{2}\right)$ is the final growth position of the root axis in a growth cycle, $\mathrm{L}$ is the lenth of the root axis, $\alpha, \beta, \gamma$ are decided by equation (9).

Growth direction of lateral root axis. The $3 \mathrm{D}$ structure of a primary root is rotated to an orthonormal coordinates (Fig.3a, b). This amount of rotation is calculated to find the position of the root in 3D space. Then the growth direction of a new lateral root on the primary root was calculated. Finally, the orthonormal coordinates was rotated back to its original orthonormal coordinates. Fig. 3a shows that the final growth direction of a new lateral root $\mathrm{P}_{2} \mathrm{P}_{3}$ was determined by the vectors $\mathrm{P}_{0} \mathrm{P}_{1}$ and $\mathrm{P}_{1} \mathrm{P}_{2}$. The three points $\mathrm{P}_{0}, \mathrm{P}_{1}$ and $\mathrm{P}_{2}$ were rotated to $\mathrm{XOY}$ of an orthonormal coordinates, the corresponding points were $\mathrm{P}_{0}, \mathrm{P}_{1}$ ' and $\mathrm{P}_{2}$, the growth direction of a new lateral root $\mathrm{P}_{2}{ }^{\prime} \mathrm{P}_{3}$ ' was denoted by a vector $v$, which can be divided to each coordinate, as $\left(v_{1}, v_{2}\right.$, $v_{3}$ ) (Fig.3b), thus,

$$
\begin{gathered}
v_{1}=|v| \cdot \sin \gamma \cdot \cos \delta \\
v_{2}=|v| \cdot \cos \gamma \\
v_{3}=|v| \cdot \sin \gamma \cdot \sin \delta
\end{gathered}
$$




$$
|v|=\sqrt{v_{1}^{2}+v_{2}^{2}+v_{3}^{2}}
$$

$|v|=$ length of vector $v, v /|v|=$ unit vector.

The required rotation is calculated by using the definition of an orthonormal axial system $x, y, z$ :

$$
\left.\begin{array}{l}
a_{1}=\left(x_{1}-x_{0}\right) /|v| \\
b_{1}=\left(y_{1}-y_{0}\right) /|v| \\
c_{1}=\left(c_{1}-c_{0}\right) /|v|
\end{array}\right\} \rightarrow \text { Unit vector } x=\left(a_{1}, b_{1}, c_{1}\right)
$$

Similarly, $\mathrm{Y}=\left(a_{2}, b_{2}, c_{2}\right), \mathrm{Z}=\left(a_{3}, b_{3}, c_{3}\right), \mathrm{X}=\left(a_{4}, b_{4}, c_{4}\right)$, Now we have the unit vectors $X, Y$ and $Z$ with their components, which form a matrix $R$ :

$$
\left(\begin{array}{l}
x \\
y \\
z
\end{array}\right)=\left(\begin{array}{l}
a_{4} b_{4} c_{4} \\
a_{2} b_{2} c_{2} \\
a_{3} b_{3} c_{3}
\end{array}\right)=R
$$

Reverse rotation and translation will bring the structure back to its original position. Vector $v$, representing the new link $\mathrm{P}_{2} \mathrm{P}_{3}$, will thereby be multiplied by the transposed matrix $R$.

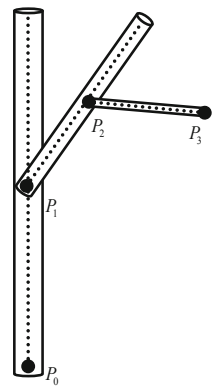

a. The determination of lateral root growth direction

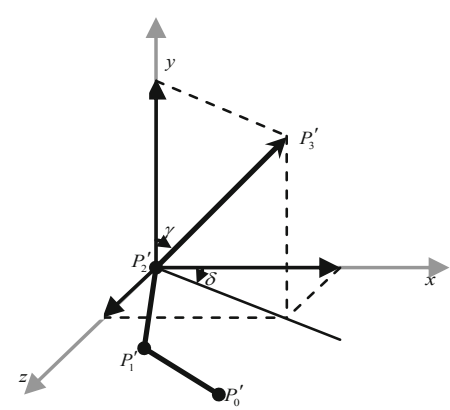

b. Structure of lateral root in orthonormal coordinates

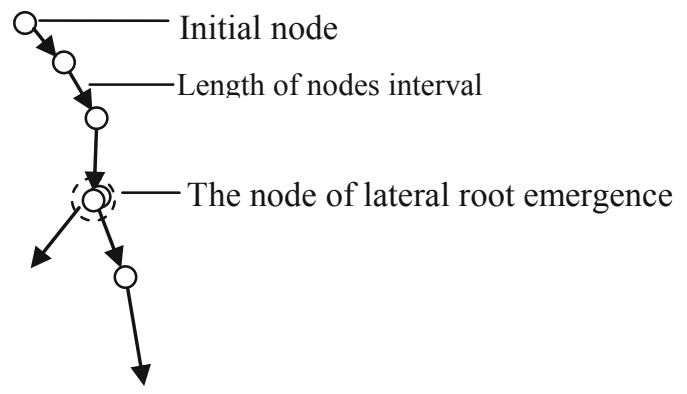

c. Root axis curve formation

Fig. 3. Growth direction of lateral root in wheat 
Root axis curve formation. The root axis was comprised of numerous root segments. The root axis grows $1{ }^{\circ} \mathrm{Cd}$ as a step. When the root axis grows a certain length, from the root axis emerges a node which produces a lateral root according the rule explained in 3.1.1. The curve of the root axis was formed in this manner of root growth, as showed in Fig.3c.

\subsection{Water Stress and Nitrogen Deficit Effect on Root Architecture}

Water stress. Wheat root architecture is influenced by soil water content. The relative growth rate was used simulate the root growth process under water stress [21].

$$
\frac{d R}{R d t}=Y_{R}(t)\left[\frac{w(t)\left(w(t)-w_{i}\right)}{w_{0}\left(w_{0}-w_{i}\right)}\right]+\alpha(t) R(t) \quad w_{i}<w(t)<w_{0}
$$

where $R(\mathrm{t})$ is the root weight, $w(t)$ is the actual soil water content, $W_{\mathrm{i}}$ is the minimum soil water content, $w_{0}$ is the critical soil water content, $\mathrm{YR}(\mathrm{t})$ is a genetic factor of the root in different developmental stages, and $\alpha(t)$ is a function coefficient of the root.

Nitrogen deficit. Low soil nitrogen content can stimulate root growth, and high soil nitrogen content would restrict root growth. According to the CERES model [22], Nitrogen deficit factor (FN) which is a value within $0-1$ can be calculated as following:

$$
\mathrm{FN}=1-(\mathrm{TCNP}-\mathrm{TANC}) /(\mathrm{TCNP}-\mathrm{TMNC}) \text {. }
$$

where TCNP, TANC and TMNC are critical, actual and minimum soil nitrogen content, respectively.

\subsection{Random Processing}

To avoid rigid root curves while using the visualization model, the root morphological parameters were randomized to enhance the effect of "shake". Based on the morphological feature parameters outputted from the wheat root morphological model, it was necessary to add a random variable between the high-low limit to realize realistic effect. The random variable was limited to a certain range, and the accuracy rating was evaluated by average value of the parameters. According to central limit theorem and large number theorem, this model constructs random variable as described in equation (15).

$$
N_{r}=\left(N_{\max }-N_{a}\right)\left|r_{1} / 2.58\right|-\left(N_{a}-N_{\min }\right)\left|r_{2} / 2.58\right|
$$

where $N_{\mathrm{r}}$ is random variable; $N_{\mathrm{a}}$ is an average of a morphological feature parameter; $N_{\max }$ is the maximum morphological feature parameter; $N_{\min }$ is the minimum morphological feature parameter; $r_{1}$ and $r_{2}$ are the random variables subject to the normal distribution based on $\mathrm{N}[0,1]$. 


\section{Visualization Model}

In order to make the visualization of roots looks more realistic, a visualization model was developed by using 3D dimensional graphic technology on the platform of VC++.net and OpenGL library, including a root axis geometric sub-model, and a texture and illumination sub-model.

\subsection{Root Axis Geometric Sub-model}

A root system is comprised of a number of root axes, the root axes can be thought of as numerous connections of truncated cones. A truncated cone has four parameters: radius of the upper $\left(R_{1}\right)$ and lower $\left(R_{2}\right)$ circles, the height $(L)$, and the direction of root axis [17] (Fig. 4a). The connection of the truncated cones should be followed by two conditions: the forepart of the truncated cone is larger than the posterior; the primary and adventitious root is thicker than lateral roots. When two truncated cones are connected, a gap appears, as showed in Fig.5a, which affects the visualization effects. A polygon grid algorithm [23] was adopted to eliminate these gaps (Fig. 5b).

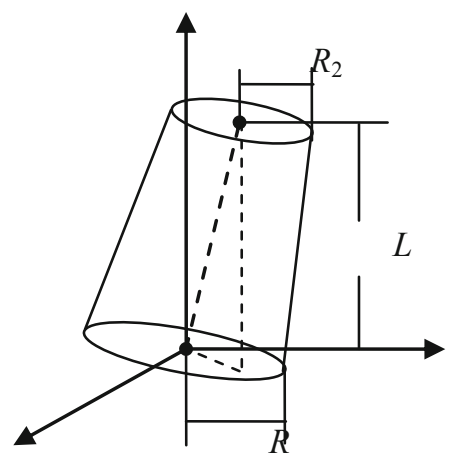

Fig. 4. The basic model of root segment
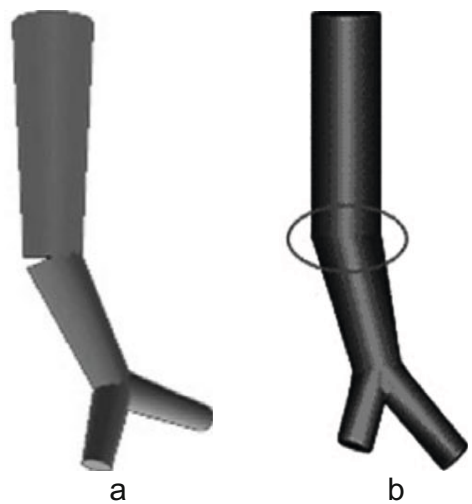

Fig. 5. Comparison of the effect of truncated cones connection

\subsection{Illumination and Texture Sub-model}

Roots are embedded in soil and grow in a dark environment, however, root structure can be looked clearly under illumination. The illumination model in the OpenGL library was adopted for simulating the illumination effect. The root surface was drawn by a texture model, which was constructed by using photos of a root at different stages with textures and integrated with the geometric model. The format of texture picture was $32 * 32$ bmp. 


\section{Visualization of Wheat Root --Example Applications}

Based on the root topological structure, linking root morphological model and visualization model, visualization of wheat root was realized by using 3D geometrical modeling techniques. Fig. 6 shows that the time-course simulation of root growth under different growth stages in wheat. The root system was from simple to complex, and the number of roots varied greatly.

An experiment was conducted to compare the visualization output in this study with field observations. The experiment was conducted at the Nanjing Agricultural University Experimental Station in Nanjing $\left(32^{\circ} 02^{\prime} \mathrm{N}, 118^{\circ} 50^{\prime}\right.$ E), China in 2009. The sowing date was 11 Nov. The cultivar Huaimai 25 was evaluated.

Fig. 7 shows the comparison between observed and simulated single root architecture. The observed root structure scanned by the WinRhizo system (Regent Instrument Inc., Canada) indicated that the observed secondary order lateral roots were less than the simulation because of mechanical damaged from manual sampling. Fig. 8 illustrates a reasonably good comparison between the visualization of root architecture and the observed root architecture at the seedling stage.

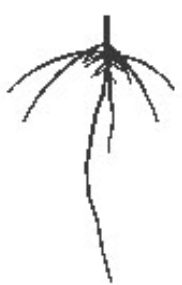

1. 6 days

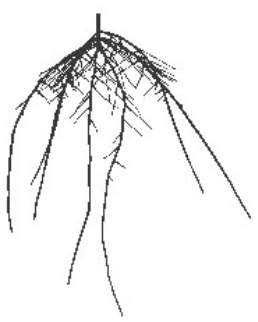

2. 15 days

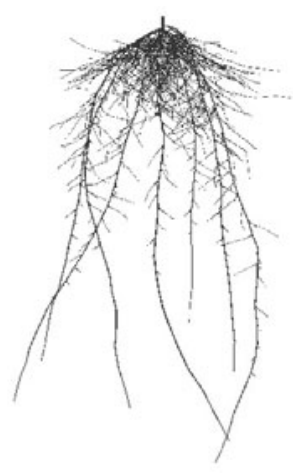

3. 25 days

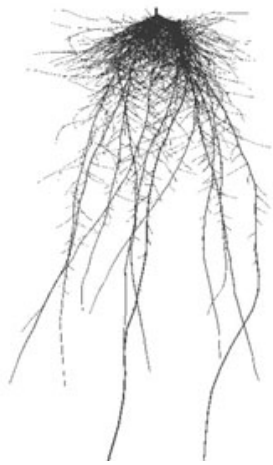

4. 35 days

Fig. 6. Visualization of root system growth under different growth stages in wheat

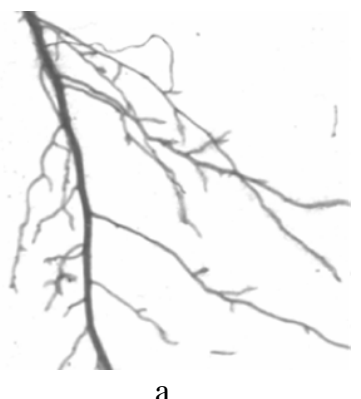

a

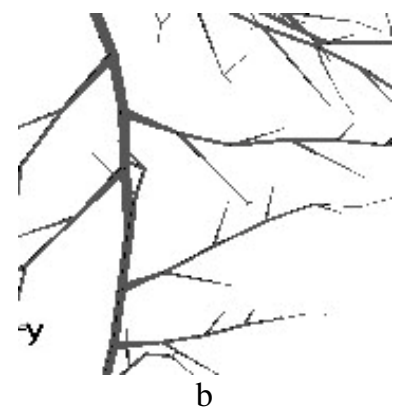

b

Fig. 7. Comparisons between an observed (a) and simulated (b) single root architecture 


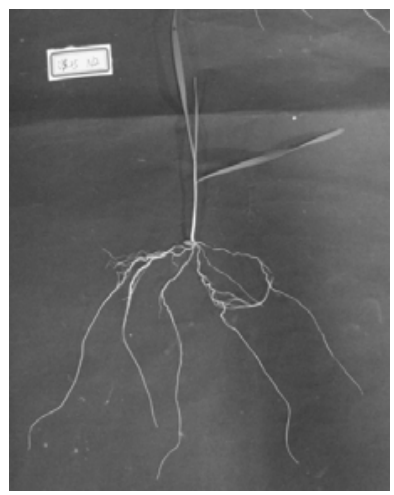

$\mathrm{a}$

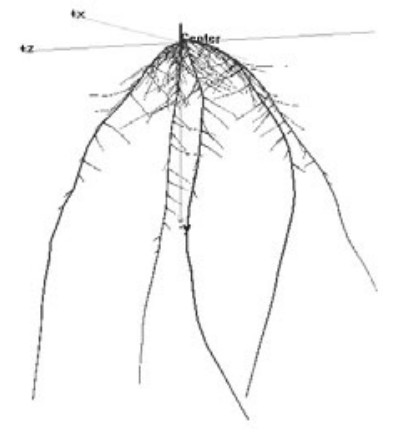

b

Fig. 8. Comparisons between observed (a) and simulated (b) root system architecture at seedling stage

\section{Discussion and Conclusions}

This paper described a root morphological model for simulating root growth and development under different conditions and a visualization model for the realistic visualization of root architecture in wheat. We aimed at the actual characteristics of root architecture in wheat. It is different from previous studies [6, 19], which aimed at a synthesis, generic root architectural model and its ability to simulate a diversity of root system architectures. Some of methods applied in those models were adopted in this study.

Other studies on visualization of root architecture used different approaches, such as fractal description [2-4], development rule [5-7], L-systems [10-11], and Greenlab $[12,13]$. These studies on wheat root either paid attention to the development or briefly described the visualization technology [5], or only observed and simulated the root morphology at the seedling stage [12].

Based on the topology of the root system in wheat, historical literature and experimental data, a root morphological model of wheat was established preliminarily using thermal time as a driving factor, including a root emergence sub-model, a root growth rate, and a root axis curve sub-model. Based on 3D visualization technology, a three-dimensional visualization model of a root axis in wheat was developed by using the platform of $\mathrm{VC}++$.net and OpenGL library, including the sub-models of geometry, texture mapping, and light rendering. Integrating the established root morphological model and the visualization model, the three-dimensional visualization of root system growth in wheat under different conditions was realized.

Roots growth and development is very complicated with a high degree of plasticity as influenced by many different factors, such as soil chemical and physical properties, climate elements, and crop genetics. Further study is needed to focus on soil, crop genotypes, and environmental conditions. 
Acknowledgments. We are thankful that the study was supported by the National Natural Science Foundation of China (30800136) and the National Basic Research Programme of China (2009CB118608). We also would like to thank Professor Albert Weiss at School of Natural Resources, University of Nebraska-Lincoln, for revising the English of this manuscript.

\section{References}

1. Manske, G.G.B., Vlek, P.L.G.: Root architecture-wheat as a model plant. In: Waisel, Y., Eshel, A., Kafkafi, U. (eds.) Plant Roots: The Hidden Half, pp. 249-259. Marcel Dekker, Inc., New York (1996)

2. Lynch, J.P., Nielsen, K.L.: Simulation of root system architecture. In: Waisel, Y., Eshel, A., Kafkafi, U. (eds.) Plant Roots: The Hidden Half, pp. 247-258. Marcel Dekker Inc., New York (1996)

3. Fitter, A.H., Stickland, T.R.: Fractal characterization of root system architecture. Funct. Ecol. 6, 632-635 (1992)

4. Ozier, L.H., Lecompte, F., Sillon, J.F.: Fractal analysis of the root architecture of Gliricidia sepium for the spatial prediction of root branching, size, and mass: model development and evaluation in agroforestry. Plant Soil 209, 167-180 (1999)

5. Van Noordwijk, M., Spek, L.Y., De Willigen, P.: Proximal root diameters as predictors of total root system size for fractal branching models. I. Theory Plant Soil 164, 107-118 (1994)

6. Diggle, A.J.: ROOTMAP - a model in three-dimensional coordinates of the growth and structure of fibrous root systems. Plant Soil, 105169-105178 (1998)

7. Pages, L., Vercambre, G., Drouet, J.L., Lecompte, F., Collet, C., Bot, J.L.: Root Typ: a generic model to depict and analyse the root system architecture. Plant Soil 258, 103-119 (2004)

8. Clausnitzer, V., Hopmans, J.W.: Simultaneous modeling of transient three-dimensional root growth and soil water flow. Plant Soil 164, 299-314 (1994)

9. Lindenmayer, A.: Mathematical models of cellular interaction in development, Parts I and II. J. Theor. Biol. 18, 280-315 (1968)

10. Prusinkiewicz, P., Lindenmayer, A.: The Algorithmic Beauty of Plants. Springer, New York (1990)

11. Shibusawa, S.: Hierarchical modeling of a branching growth root system based on L-system. Acta Hort. 319, 659-664 (1992)

12. Daniel, L., Andrea, S.: Root growth simulation using L-system. In: Handlovičová, A., Frolkovič, P., Mikula, K., Ševčovič, D. (eds.) ALGORITMY 2009 18th Conference on Scientific Computing Vysoké Tatry, pp. 313-320. Slovak University of Technology, Bratislava (2009)

13. Zhang, W.P., Guo, Y., Li, B.G.: Development and application of three-dimensional growth model of root system in wheat seedling. Scientia Agricultura Sinica 39(11), 2261-2269 (2006) ( in Chinese)

14. Zhang, W.P., Li, B.G.: Three-dimensional model simulating development and growth of cotton root system. Journal of System Simulation 18(1), 283-286 (2006) ( in Chinese)

15. Ma, Y.X.: Wheat Roots. China Agriculture Press, Beijing (1999) (in Chinese)

16. Klepper, B.: Root and shoot development in winter wheat. Agron. J. 76, 117-122 (1984)

17. Yan, M.C., Cao, W.X., Luo, W.H., Wang, S.H.: A simulation model of above-ground organ formation in wheat. Acta Agronomica Sinica 27(2), 222-229 (2001) ( in Chinese) 
18. Zhang, B.G., De Reffye, P., Liu, L., Kang, M.Z., Li, B.G.: Analysis and modeling of the root system architecture of winter wheat seedlings. In: Hu, B.G., Marc, J. (eds.) Plant Growth Modeling and Applications: Proceedings-PMA03: 2003 International Symposium on Plant Growth Modeling, Simulation, Visualization and Their Applications, Beijing, China, October 13-16, pp. 321-328. Tsinghua University Press/Springer, Beijing/ Heidelberg (2003)

19. Lynch, J.P., Nielsen, K.: SimRoot: Modeling and visualization of root systems. Plant Soil 188, 139-151 (1997)

20. Chen, J.H., Lieth, D.: A two-dimentional, dynamic model for root growth distribution of potted plants. J. Amer. Soc. Hort. Sci. 118, 181-187 (1993)

21. Mech, R., Prusinkiewicz, P.: Visual models of plants interacting with their environment. In: Fujii, J. (ed.) Computer Graphics Proceedings. Annual Conference Series, pp. 397-410. ACM SIGGRAPH, New York (1996)

22. Godwin, D.C., Singh, U.: Nitrogen balance and crop response to nitrogen in upland and lowland cropping system. In: Tsuji, G.Y., Hoogenboom, G., Thornton, P.K. (eds.) Understanding Options for Agricultural Production, pp. 55-77. Kluwer Academic Publishers, Dordrecht (1998)

23. Lluch, J., Vivo, R., Monserrat, C.: Modelling tree structures using a single polygonal mesh. Graph. Models 66, 89-101 (2004) 